\title{
Technique Of Research Uncertainty Dynamic Measurements Of Vibration Acceleration Of Rotating Machines
}

\author{
O. M. Vasilevskyi ${ }^{1}$, P. I. Kulakov ${ }^{1}$, V. M. Didych ${ }^{2}$ \\ ${ }^{1}$ Vinnytsia National Technical University, 95 KhmelnitskoyeShose, Vinnytsia, 21021, Ukraine, \\ ${ }^{2}$ Vinnytsia National Medical University named after M. I. Pirogov
}

\begin{abstract}
Aabstract: Developed methodology for estimation of dynamic measurement uncertainty, which corresponds to the international standards for evaluating the quality of measurements - the concept of uncertainty. It is proposed to estimate the uncertainty of dynamic measurements on the basis of the frequency characteristic measuring means and the spectral function of the input signal. This method was tested in the evaluation of the dynamic measurement of vibration of rotating machines that helped confirm its correctness and efficiency. It is found that the maximum value of the dynamic uncertainty vibration acceleration measurement of $0,05 \mathrm{~m} / \mathrm{s}^{2}$ for an observation time of $300 \mathrm{~s}$ and the nominal value signal of vibration acceleration $0.35 \mathrm{~m} / \mathrm{s}^{2}$ at a frequency of 6 $\mathrm{kHz}$.
\end{abstract}

Keywords: dynamic uncertainty of measurement devices, quality assurance of dynamic measurements, spectral function, frequency characteristic, vibration acceleration.

\section{Introduction}

Experiments conducted using Measurement Means (MM) under dynamic conditions are becoming more common in many fields including scientific research, technology, manufacturing industry, commerce, and medicine. Dynamic measurements are in the first instance concerned with the study of the conformity of the path of the physical processes in subjects under investigation. As a result, the role of such measurements is particularly significant, firstly in the areas of science related to the investigation of the structure of matter, the analysis and synthesis of new substances and materials, where the study of takes place under experimental conditions, and secondly, in the fields of engineering, especially in manufacturing and medicine, which are which characterized by the creation of new technological processes and the testing of new MM using high precision equipment.

When reporting on the results of dynamic measurements, it is necessary to provide a quantitative assessment of the quality of the experiment in order that its reliability may be correctly appraised [1-5]. Without such a reference value, the results of dynamic measurement can neither be compared with other equivalent studies, nor with standard reference values. It is therefore necessary to develop a uniform and understandable assessment methodology of the quality characteristics of dynamic measurements.

In this context, is necessary to take into account the fact that during dynamic measurement there is always a transition period during the operation of the MM, during which the output signal of the MM changes significantly over time. This set of circumstances may be explained given the inertial properties of the MM, because they comprise, on the whole, of components with different masses and springs, capacitance and inductance or other inertial elements, that lead to the emergence of dynamic uncertainty. This leads to the fact that the means of measuring the conversion equation that maps its statics is unacceptable in a dynamic mode. In this case, we need to use differential equations that describe the dynamic relationship of the output, $y(t)$, and the input $\mathrm{x}(\mathrm{t})$ values of measuring instruments [6].

Given the above, it is necessary to develop a single approach to the expression of dynamic uncertainty in measurement means, which would meet international requirements that apply to the evaluation of the characteristics of the quality of measurements, and this is seen as the core scientific problem in the field of metrology.

The objective in writing this paper: is the development and mathematical description of a new approach to the expression of the dynamic uncertainty of measurement means, that would allow us to take into account the inertial properties of the measurement means and the measurement signal passing through it, which will ensure a unified estimation of dynamic measurements in accordance with existing regulations in metrology, even if produced in different countries, different laboratories and different metrological organizations. 


\section{Analysis of the status of research and publications}

In drawing up relevant differential equations, input signals are recorded on the right, i.e. the reason that led the MM to function, while the left side of the differential equation, describes the output signal (or response of the MM), and for linear transducers, it is written in the form [6,7]

$$
\sum_{i=0}^{n} a_{i} y^{i}(t)=\sum_{k=0}^{m} b_{k} x^{k}(t)
$$

where $x(t), y(t)$ are respectively the input and output values; $i, k$ are the order derivatives; and $\mathrm{a}, \mathrm{b}$ are the coefficients that characterize the properties of the MM.

To express the differential equation in the area of frequency, the differentiation symbol $j \omega$ may replace $d / d t$ as the time coordinate, and then the equation (1) takes the form:

$$
\frac{y(j \omega)}{x(j \omega)}=S_{o} \frac{b_{m}(j \omega)^{m}+b_{m-1}(j \omega)^{m-1}+\ldots+1}{a_{n}(j \omega)^{n}+a_{n-1}(j \omega)^{n-1}+\ldots+1},
$$

or

$$
y(j \omega)=H(j \omega) x(j \omega) \text {, }
$$

where $y(j \omega), x(j \omega)$ are respectively the spectral functions of the input and output measurement signal; $\mathrm{S}_{0}=b_{0} / a_{0}$ is the static sensitivity, i.e. the sensitivity to the constant input value (when $j \omega=0$ ); $H(j \omega)$ is the transfer function of the MM or operational sensitivity.

The most typical properties of the MM are dynamic characteristics, which are described by differential equations of the first or second order, although in some cases, the third or higher order [4-7].

Information about the dynamic characteristics should be found in the regulatory and technical documentation of the MM, although if data is not available, it can be obtained on the basis of a priori data on the MM.

To express the experimental uncertainty of the results of dynamic measurements, it may be convenient for practical use to refer to the frequency characteristics of the measuring means $[4,8]$, listed in Tab. 1.

\section{Table 1}

\begin{tabular}{|l|l|}
\hline $\begin{array}{l}\text { Frequency characteristics of the MM } \\
\text { where } K \text { is the transmission coefficient }\end{array}$ & Typical Units \\
\hline$H(j \omega)=\frac{K}{1+j \omega \tau}$ & Aperiodic (temperature transducer) \\
where $\tau$ is the time constant determined by the parameters of the MM & \\
\hline$H(j \omega)=\frac{K}{j \omega}$ & Integrated (integrated amplification) \\
\hline$H(j \omega)=K(1+j \omega \tau)$ & Forcing (differential amplification) \\
\hline$H(j \omega)=\exp (-j \omega \tau)$ & Delay (analog-to-digital converters) \\
\hline$H(j \omega)=\frac{K}{1+j \omega \tau_{1}-\omega^{2} \tau_{2}^{2}}=$ & Oscillating (electromechanical transducers) \\
$=\frac{K}{1+2 j \omega \beta \tau-\omega^{2} \tau^{2}}$ & \\
\hline
\end{tabular}

It is also known that the existing international experience in the concept of evaluation and expression of measurement uncertainty [1] does not describe how to undertake estimation of dynamic uncertainties in the performance of metrological works (or experiments in dynamic modes of MM). [1] only makes it apparent that in existence there are ways of estimation as demonstrated by type A and type B, and in addition ways to demonstrate uncertainties, which may be standard, combined or enhanced. The definitions of these uncertainties are given in [1]. A well-known approach, as investigated in the papers [4-6], is that dynamic uncertainty is calculated as a standard uncertainty of type B, itself determined by the dynamic error value divided by the square root of 3 (assuming a uniform distribution law). 
Using classical theory in the measurement of dynamic error in the expression of dynamic uncertainty is unacceptable, given the concept of measurement uncertainty expression, which, as set out in the international standard [1], is moving away from the concept of measurement error, as such, which does not use known values, and cannot have absolute values. This is as opposed to measurement uncertainty, which can be evaluated, and for a particular measurement result is not a single value, but has an infinite number of values, which are scattered around the result.

Consequently, there is a need to develop a new approach to the expression of dynamic uncertainty that can be evaluated without using the classic dynamic errors used in error theory.

\section{The presentation of the base material}

If the equation of the measuring transmitter is

$Y=K_{C} X$,

where $X$ - a physical quantity to be measured (input signal); $K_{C}$ - transformation coefficient of the measuring device; $Y$-measurement result (output signal).

Then mathematical expectation of the input signal equals to $M[X]$, and mathematical expectation of the output signal will equal

$\mathrm{M}[\mathrm{Y}]=\mathrm{K}_{\mathrm{C}} \mathrm{M}[\mathrm{X}]$,

where $M[Y]$ and $M[X]$ - mathematical expectations of the output and input signals of measuring device correspondingly.

Spectral density of the input signal $X(t)$ has the equation [4]

$H_{X}(\omega)=\lim (2 T)^{-1}|X(j \omega)|^{2}$ when $T \rightarrow \infty$,

where $X(j \omega)$ - fourier image, received by substitution of the meanings on $j \omega$ in the operator image $X(s) ; T-$ observation time; $\omega=2 \pi f$.

Similarly the equation for spectral density of output signal can be represented as

$H_{Y}(\omega)=\lim (2 T)^{-1}|Y(j \omega)|^{2}$ when $T \rightarrow \infty$.

Images ratio of output and input meanings results in the equation of measuring device transfer function $[4,7]$

$K_{C}(s)=\frac{Y(s)}{X(s)}=\frac{\sum_{k=0}^{m} B_{k} s^{k}}{\sum_{q=0}^{n} A_{q} s^{q}}$,

where $Y(s), X(s)$ - operators images of output $Y(t)$ and input $X(t)$ signals correspondingly; $k, q$ - derivative orderfrom $Y$ и $X$ correspondingly; $A_{q}, B_{k}$ - coefficients of differential equation.

Therefore it follows that [7]

$$
H_{Y}(\omega)=\left|K_{C}(j \omega)\right|^{2} H_{X}(\omega) \text {, }
$$

where $K_{C}(j \omega)$ - frequency characteristics of measuring transformer.

We can determine the uncertainty of output signal on dynamic measurements as a square root from integral of output signal spectral density according to all available frequencies [7]

$u_{D}=\pi^{-1 / 2}\left(\int_{0}^{\infty}\left|K_{C}(j \omega)\right|^{2} H_{X}(\omega) d \omega\right)^{1 / 2}=\pi^{-1 / 2}\left(T^{-1} \int_{0}^{\infty}\left|K_{C}(j \omega)\right|^{2}|X(j \omega)|^{2} d \omega\right)^{1 / 2}$,

where $\left|K_{C}(j \omega)\right|$ - modulus of measuring device frequency characteristics being used for dynamic measurement.

Modulus of measuring device frequency characteristic is determined by the formula

$\left|K_{C}(j \omega)\right|=\left(a^{2}(\omega)+b^{2}(\omega)\right)^{1 / 2}$,

where $a(\omega), b(\omega)$ - real and imaginary parts of frequency characteristic correspondingly $K_{C}(j \omega)$ [9].

Spectral function of the input signal $X(j \omega)$ is linked to its time function $X(t)$ by Laplace equation

$$
X(j \omega)=\int_{0}^{\infty} X(t) e^{-j \omega_{0} t} d t
$$

where $\omega_{0}$ - circular frequency of input signal [4-9].

Infinity sign can be substituted by summary observation time $T$ when time interval is final. 
So the uncertainty introduced by the limited capacity of the measuring device on dynamic measurements can be evaluated on the basis of the model equation of input signal spectral function and frequency characteristics of the used device by the formula (9).

\section{The method of dynamic vibration acceleration measurement uncertainty estimation}

When measuring thevibration acceleration of operating mechanisms acting on the basis of the accelerometer mechanical vibrations lead to the fact that the piezo is affected by dynamic force that is the product of the acceleration of the seismic mass to the appropriate weightmultiplication. Piezoelements generate an electrical charge proportional to the current dynamic force for it. Seismic mass of the accelerometer virtually unchanged, which means that the electric charge that occurs at the electrodes of the piezoelements is proportional to the acceleration of the masses. Since the amplitude and phase of accelerating seismic mass in a broad frequency range are identical to the amplitude and phase of accelerometer bases acceleration, the overall electric charge, given to the latter is proportional to the acceleration of its foundations and therefore acceleration of the mechanical vibrations of the objectsurfaces, to which accelerometer is fixed [10].

Differential equations describing the dynamic relationship of input and output variables of acceleration measuring channel has the form

$m \frac{d^{2} X_{s}(t)}{d t^{2}}+c \frac{d X_{s}(t)}{d t}+k X_{s}(t)=F(t)$,

where $F(t)=F_{0} \sin \left(\omega_{0} t\right)$ is harmonic forced oscillations power of the object surfaces(input value); $F_{0}$ is power amplitude; $\omega_{0}$ is angular frequency of forced power; $X_{s}(t)$ is mechanical vibrations of the inertial mass; $m$ is accelerometer mass; $c$ is damping parameter; $k$ is piezoelements equivalent stiffness [10].

Let us write the differential equation (12) in the form of commonexpretion

$\frac{d^{2} X_{s}(t)}{d t^{2}}+2 h \frac{d X_{s}(t)}{d t}+h_{k}^{2} X_{s}(t)=\frac{F_{0}}{m} \sin \left(\omega_{0} t\right)$,

where $h=c /(2 m)$ is damping coefficient; $h_{k}=\sqrt{k / m}$ is critical damping coefficient.

The transfer function of the measuring tool has a look

$$
H(s)=\frac{K_{M M}}{s^{2}+2 h s+h_{k}^{2}},
$$

where $K_{M M}$ is proportionality factor of vibration acceleration measuring channel.

Turning to the frequency domain and separating the real and imaginary parts, we obtain an expression of the module of frequency measuring vibration acceleration characteristics

$$
\left|K_{C}(j \omega)\right|=\left|\frac{K_{M M}}{(j \omega)^{2}+2 h(j \omega)+h_{k}^{2}}\right|=\left[\frac{K_{M M}}{\omega^{4}-2 \omega^{2} h_{k}^{2}+4 \omega^{2} h^{2}+h_{k}^{4}}\right]^{1 / 2} .
$$

Input signal $F_{0} m^{-1} \sin \left(\omega_{0} t\right)$ of the vibration acceleration has image

$$
X(j \omega)=j \omega_{0} F_{O}\left(\omega_{O}^{2}+(j \omega)^{2}\right)^{-1} m^{-1},
$$

where $\omega_{0}$ is cyclic frequency of input vibration acceleration signal, which ranges from 6 to $10 \mathrm{kHz}$, that is, its minimum value is $18,849.5 \mathrm{rad} / \mathrm{s}$, and the maximum value is $31415.9 \mathrm{rad} / \mathrm{s}$.

The module of input vibration acceleration image described by

$|X(j \omega)|=\omega_{0} F_{0}\left(\omega_{0}^{2}-\omega^{2}\right)^{-1} m^{-1}$.

From the literature $[10-12]$, it is known that the amplitude of the forced harmonic force $F_{0}$ is $0,3 \mathrm{~mm}$, which corresponds to $3 \cdot 10^{-4} \mathrm{~m}$. Weight accelerometer is $\mathrm{m}=40 \mathrm{~g}$, corresponding to $4 \cdot 10^{-2} \mathrm{~kg}$. Damping parameter $c$ for piezoelectric accelerometers is 0,5 , equivalent stiffness piezoelements is $\mathrm{k}=2$, the observation time is $\mathrm{T}=300 \mathrm{~s}$. The proportionality factor (gain) $K_{M M}$ of vibration acceleration measuring channel is $10^{5}$.

Substituting the found values of frequency characteristics module (15) and input signal image (17) in equation

(9) we obtain an expression for the estimation of measurement uncertainty dynamic vibration acceleration 
$u_{D}=\pi^{-1 / 2}\left(T^{-1} \int_{0}^{\infty} \frac{K_{M M} \omega_{0}{ }^{2} F_{0}{ }^{2}\left(\omega_{0}^{2}-\omega^{2}\right)^{-2} m^{-2}}{\omega^{4}-2 \omega^{2} h_{k}^{2}+4 \omega^{2} h^{2}+h_{k}^{4}} d \omega\right)^{1 / 2}$.

For the solution of equation (18) mathematical package Maple 12 was used. Substituting in the expression (18) the mentioned above factors there was obtained the numeric value of measurement uncertainty dynamic acceleration, which is $0,033 \mathrm{~m} / \mathrm{s} 2$ at the frequency of the input signal $10 \mathrm{kHz}$ and acceleration time of observation $\mathrm{T}=300 \mathrm{~s}$. If the observation time increases to $600 \mathrm{~s}$ at the same frequency as the input vibration acceleration, the value of dynamic uncertainty reduces to $0,023 \mathrm{~m} / \mathrm{s} 2$. Rated motor acceleration signal from electrospindles is $0,35 \mathrm{~m} / \mathrm{s} 2[10-12]$.

At the minimum frequency of the vibration acceleration input signal which is $6 \mathrm{kHz}$ and at the time of observation with $300 \mathrm{~s}$, the value of dynamic uncertainty is $0,049 \mathrm{~m} / \mathrm{s} 2$. If the observation increases to $600 \mathrm{~s}$ with the frequency of the input signal vibration acceleration $6 \mathrm{kHz}$, the value of dynamic uncertainty is reduced to $0,035 \mathrm{~m} / \mathrm{s} 2$ (nominal motor vibration acceleration signal from electric spindel is $0,35 \mathrm{~m} / \mathrm{s} 2$ ). Dynamic uncertainty changes characteristics depending on the observation time T (from 10 to 1500 s) are obtained using mathematical package Maple 12 and presented in Figure 1 at minimum $6 \mathrm{kHz}$ and $10 \mathrm{kHz}$ maximum values of the input signal of frequency vibration acceleration.

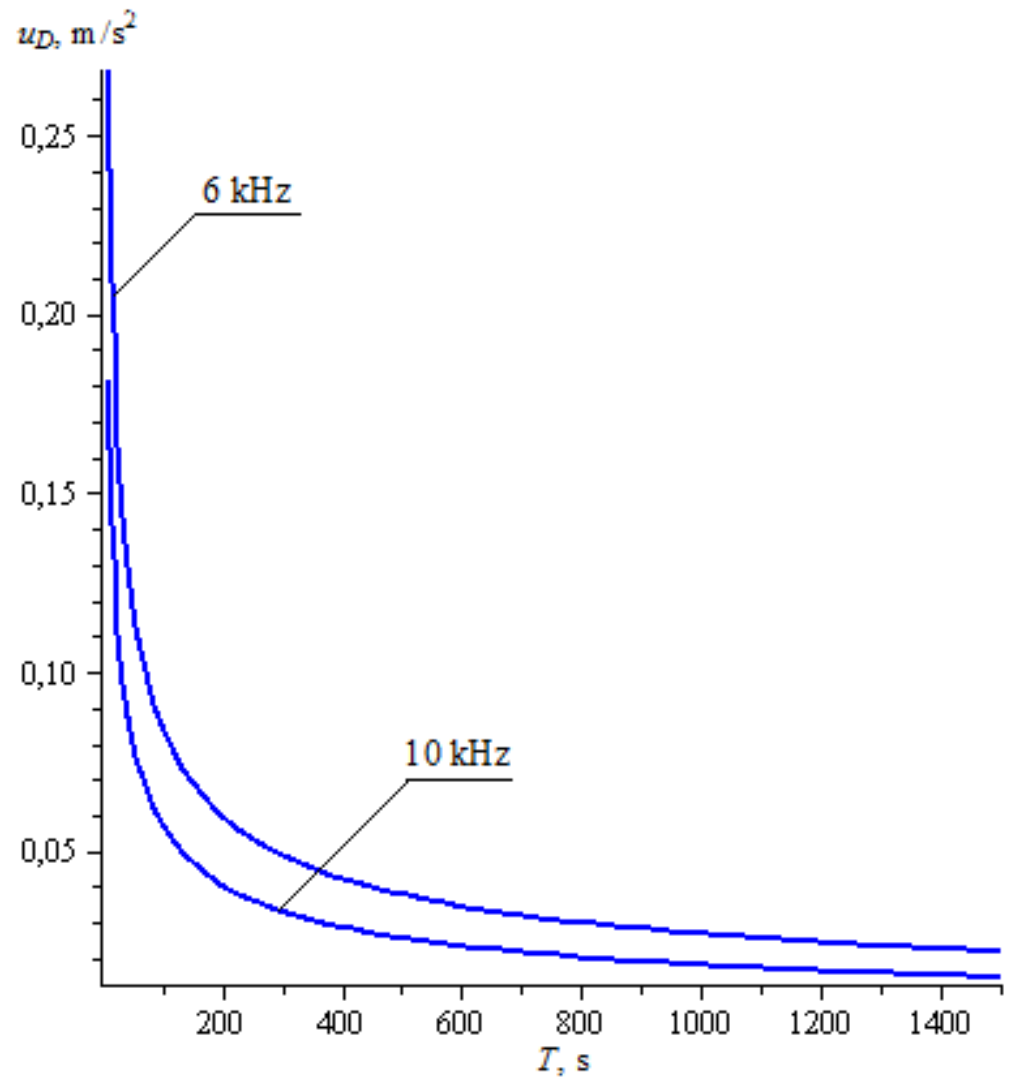

Figure 1

Thus, on basis of the proposed method of evaluation of dynamic measurements uncertainty there has been estimated the uncertainty of vibration acceleration dynamic measurement of electric motor based on mathematical models of the spectral function of the input acceleration and frequency response of measuring channel acceleration.It resulted in the opportunity, together with the values of the standard uncertainty type A, standard uncertainty type B that due to the limited properties of measuring transducers, which are part of measuring channel acceleration, consider the value of dynamic uncertainties in assessing the combined (total) measurement uncertainty acceleration.

As a result of studies it has been found out (Fig. 1), that the smaller the observation time of vibration acceleration signal of a moving object, is the bigger is value of dynamic measurement uncertainty. Therefore, to 
reduce the impact of dynamic measurement uncertainty in the assessment of the measurement result of vibration acceleration is necessary to increase observation time of at least $300 \mathrm{~s}$.

\section{Conclusions}

The technique of estimation of dynamic measurements uncertainty allows to calculate the value of dynamic uncertainties, taking into account that international requirements for performance evaluation of the accuracy of measurement - the concept of uncertainty. It helps to ensure traceability and enables comparison of the results of dynamic measurements made by different measurement and testing laboratories of the leading countries. The method was tested in the assessment of dynamic measurement uncertainty of electric motor vibration acceleration with electric spindel, which confirmed its validity and effectiveness.

\section{References}

[1]. ISO/IEC Guide 98-1:2009 «Uncertainty of measurement - Part 1: Introduction to the expression of uncertainty in measurement». Geneva (Switzerland): ISO. - 2009. - 32 p. (Eng).

[2]. IEC GUIDE 115-2007 «Application of uncertainty of measurement to conformity assessment activities in the electrotechnical sector». - Geneva (Switzerland): IEC. - 2007. - 54 p. (Eng).

[3]. ISO/IEC 17025:2005 «General requirements for the competence of testing and calibration laboratories». - Geneva (Switzerland): ISO. - 2005. -28 p. (Eng).

[4]. Eichstädt S. Analysis of Dynamic Measurements. Evaluation of dynamic measurement uncertainty. - Berlin: Frankenberg, 2012. 87 p. (Eng).

[5]. Esward T. J., Elster C., Hessling J. P. Analysis of dynamic measurements: new challenges require new solutions // XIX IMEKO World Congress on Fundamental and Applied Metrology. - 2009. - PP. 2307 - 2310. (in Portugal). (Eng).

[6]. Elster C., Eichstädt S., Link A. Uncertainty evaluation of dynamic measurements in line with GUM // XIX IMEKO World Congress on Fundamental and Applied Metrology. - 2009. - PP. 2311 - 2314. (in Portugal).

[7]. Vasilevskyi O. M. A frequency method for dynamic uncertainty evaluation of measurement during modes of dynamic operation // International Journal of Metrology and Quality Engineering. - 2015. - Vol. 6. - Number 2. - $202 . \quad-$ http://dx.doi.org/10.1051/ijmqe/2015008. (in United Kingdom).

[8]. Vasilevskyi O., Kucheruk V., Kurytnik I. An approach to the evaluation of dynamic uncertainty in measurement using non-statistical techniques // Pomiary. Automatyka. Kontrola. - 2014. - Vol. 60. - Number 11. - PP. 997-1001. (in Polish).

[9]. Vasilevskyi O. M. Assessment of the uncertainty of dynamic measurements // Visnyk Vinnytskogo politekhnichnogo instytutu. 2011. - № 3. - C. 9 - 13. (Ukr.).

[10]. Vasilevskyi O. M., Podzharenko V. O. Analysis of dynamic metrological characteristics of measuring vibration transducer // Nauk. pr. IV Mizhnarodnoy naukovo-techn. konf. [„Metrologiya ta vymiryvalna technika”]. - Kharkiv: NNTS «Instytut metrologii», 2004. - T. 2. - P. 130 - 132. (Ukr.).

[11]. Podzharenko V. O., Vasilevskyi O. M., Sevastyanov V. M. Evaluation of static metrological characteristics of measuring vibration channel // Ukrainskyy metrologichnyy zhurnal. - 2005. - № 2. - P. 60-65. (Ukr.).

[12]. Vasilevskyi O. M. Information-measuring system for automatic control of asynchronous rotation and vibration diagnostics of electromechanical systems // Vibratsiya mashin: izmerenie, snizhenie, zaschita. - Donetsk. - 2005. - № 1. - P. 29-32. (Ukr.). 